\title{
Evaluation of the effectiveness of typical casing treatments for a low-speed compressor by an integral method
}

\author{
NAN Xi ${ }^{1}$, MA Ning $^{1,2}$, LI JiChao $^{1}$, LIU Le $^{1}$, LIN Feng $^{1}$ \\ ${ }^{1}$ Key Laboratory of Advanced Energy and Power, Institute of Engineering Thermophysics, \\ Chinese Academy of Sciences, Beisihuanxi Road 11, Beijing 100190, China \\ ${ }^{2}$ University of the Chinese Academy of Sciences, Beijing 100049, China
}

\begin{abstract}
[The abstract has been totally revised]A novel integral method is proposed to quickly assess the effectiveness on stability improvement with end-wall casing treatments. With this low-cost method, efficacies for various casing treatment configurations can be evaluated instead of calculating the entire performance curves, which can be used in place of expensive and costly experiments. The underlying mechanism for this approach is based on the hypothesis that the spike stall precursors can be triggered by the forward spillage of the rotor tip leakage flow, and the onset condition of such a spillage is determined by the axial momentum balance within the rotor tip region. Based on the simulation, a series of control volumes are set at the rotor tip region in order to catch the axial momentum balance between the incoming main flow and the reversed tip leakage flow. $\mathrm{Cu}-$ mulative axial momentum distributions for these control volumes named as "bell-shaped curves" are presented to evaluate the effectiveness of different configurations. The axial location of the bell curve peak indicates the time- and spatial- averaged interface position between the main flow and tip leakage flow, which moves upstream during throttling. Three types of typical casing treatments: multiple circumferential grooves, skewed axial slots and self-injection configurations for a low-speed in-house compressor are evaluated by their bell-shaped curves. Among these configurations, the skewed axial slots has the most downstream of the peak, followed by the multiple-grooves, while the self-injection configuration shows the least. Based on an existed experimental result for a double-groove configuration, the effectiveness of the three studied casing treatments are predicted by their locations of the bell-shape curve's peak without simulating the entire performance curves. The assessments are then validated by experiments.
\end{abstract}

Control volume method, stall margin improvement (SMI), casing treatment, axial momentum, bell-shaped curves

\section{NOMENCLATURE}

$\begin{aligned} \text { Abbreviation } & \\ \mathrm{NS} & =\text { Near stall point } \\ \mathrm{MF} & =\text { Main flow } \\ \mathrm{TLF} & =\text { Tip leakage flow } \\ \mathrm{SMI} & =\text { Stall margin improvement } \\ \mathrm{SC} & =\text { Smooth casing } \\ \mathrm{CT} & =\text { Casing treatment } \\ \mathrm{CS} & =\text { The casing surface } \\ \mathrm{BT} & =\text { The bottom surface } \\ \mathrm{Z}- & =\text { The left surface of the control volume } \\ \mathrm{Z}+ & =\text { The right surface of the control volume } \\ \text { Symbols } & \\ Z & =\text { The axial direction }\end{aligned}$

$\begin{aligned} V z & =\text { Axial velocity, }(\mathrm{m} / \mathrm{s}) \\ M z & =\text { Axial momentum, }(\mathrm{N}) \\ C_{a z} & =\text { Tip axial chord, }(\mathrm{mm}) \\ Z_{b p} & =\text { Normalized axial location of bell curve's peak }\end{aligned}$

Greek letters

$\rho \quad=$ Density, $\left(\mathrm{kg} / \mathrm{m}^{3}\right)$

$\varphi \quad=$ Mass flow coefficient

$\phi=\frac{V}{U} \quad V:$ Inlet flow velocity, $(\mathrm{m} / \mathrm{s})$

$U:$ Mid span rotating speed, $(\mathrm{m} / \mathrm{s})$

$\psi \quad=\quad$ Pressure rise coefficient $\psi=\frac{P_{2, s}-P_{1, t}}{\frac{1}{2} \rho U^{2}} \quad P_{1, t}:$ Total pressure at inlet, $(\mathrm{Pa})$ $P_{2, s}:$ Static pressure at outlet, $(\mathrm{Pa})$ 


\section{Introduction}

Casing treatments (CT) are widely investigated as the passive method to favourably alter the stall point of axial compressor. Maintaining considerable stall margin extension with minimal efficiency decrement, while also addressing part speed operability are the design concepts in this topic [1]. Experimental and numerical efforts have been dedicated to develop a more complete understanding on why the casing treatments are useful and how they can work better [2-4]. Two of the more successful designs examined are the axial slot type casing treatment and the circumferential groove type casing treatment. Fujita and Takata [5] investigated the effectiveness of the axial skewed slot, circumferential groove and the axial slot. Among those tested configurations, the skewed slots showed the highest stall margin improvement and the slot-type casing treatments showed higher efficiency penalty than circumferential grooves.

Although huge processes have been achieved, it is still a tough task for designing a satisfactory casing treatment configuration. For a given CT, the effectiveness can be different when it is applied in various circumstances. Even for the same compressor, stall margin extensions are usually irregular at different rotating speed. The modern powerful computers make it possible to utilize numerical simulations, especially with the advanced high-fidelity computational models, to explore in details the flow structures and the stalling mechanism with and without casing treatments [6]. However, due to the complexity of the stall phenomena, the mechanism of the stall margin improvement by CT is still not completely clear. Also it still remains a big challenge to accurately and reliably predict the CTs' effectiveness before actually testing them. For the compressor industry, the most common design approach is to establish a database of casing treatments through extensive experimentations, which can be very time-consuming and costly.

[This paragraph has been totally re-written]Facing this difficulty, the motivation of this paper is to propose a method to quickly assess the effectiveness of various casing treatments. The philosophy of this paper is based on such insightful research results done previously in our research group as wells as by others in the community, but NOT to get involved into these flow details this time. Instead, we are trying to develop a method to pre-screen the casing treatments for engineering designers. If the design scope can be quickly narrow down from massive candidates by a low-cost CFD tool without turning into more expensive experimental and CFD approaches, it will be beneficial at the early design stage.

Aiming at quickly pre-screeninga variety of groove configurations and providing a few more preferable ones for further detailed test, the authors have previous [7] developed a control volume method to assess the efficacy of the circumferential grooves. Based on the understanding on the stalling mechanism of tip-critical axial compressors is the MF/TLF interface spilling from the leading edge which is determined by the axial momentum distribution of the tip region. The idea of using MF/TLF interface to judge the stability comes from the hypothesis that for tip critical compressors with spike stall inception, the forward spillage of the tip leakage flow is a necessary condition for spike formation [8]. This hypothesis has been studied by many researchers [9] and validated in many experiments $[10,11]$. Cameron et al. [11] carried out rotor-casing oil flow pattern experiments in a transonic compressor. On a transparent window on the compressor casing, this interface appeared as a straight line in the absolute reference frame, see Figure 1. Considering this line as the trace of the zero axial wall shear stress, they proposed a 1-D model of the tip gap flow, and pointed out that the MF/TLF interface was driven by the tip axial momentum balance between the main flow and the tip leakage flow. As the compressor approaches to stall, the axial momentum of the main flow decreases and that of the tip leakage flow increases. The zero axial wall shear line moves upstream. Stall happens once this line spills over the rotor leading edge.

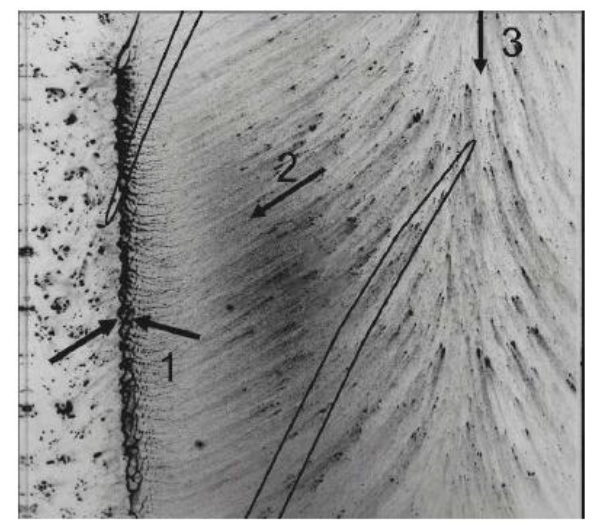

Figure.1 MF/TLF interface projection viewed as the zero axial wall shear on casing a transonic compressor in Ref. [10].

From these perspectives, the axial momentum distribution is closely related to the MF/TLF interface, which could be a rational indicator to indentify the stability of tip-critical compressor. The core idea for the method proposed in this current approach is to the interface can be down-stream pushed by the effective casing treatment, which can be indicated by a re-distributed axial momentum. To determine the final balance between the main flow and the TLF, the axial momentum of the rotor tip region is acquired by utilizing the control volumes whose depth is deep enough to contain the entire tip leakage effects. The cumulative axial momentum distribution of the tip region named as the bell-shape curve is developed to describe the location of the interface and employed to assess the effectiveness. The bell-shape curve is pushed downstream for the grooved 
casing compared to the solid wall, which indicates the MF/TLF interface is down-pushed by the grooves and the stalling delays. Rather than predicting the exact stall point for each casing treatment configuration via computation, their effectiveness is compared at the near stall point of the smooth casing using the bell-shaped curves. This method provides a different perspective for quickly assessing the grooves based on the associated flow physical insight.

This integral method provided by the authors is validated to be potential in evaluating the effectiveness of groove type casing treatment. However, to facilitate this integral method for a wider application scope, at least three objectives should be accomplished:

1) Verify this method can be extended into general end-wall treatments, such as slot-type casing treatments and self-injection casing treatments;

2) Discuss the accuracy of this integral control volume method;

3) Preliminary quickly estimate the stall margin improvement (SMI) for the various casing treatments by this current method without simulating the entire performance curves.

This paper is then organized as following: First, we will introduce the studied examples. A low-speed in-house compressor and three different casing treatment configurations are introduced. After a brief introduction of the control volume setup, the bell-shaped curves and its relation with compressor stability are discussed in detail. Then, the studied casing treatment configurations are evaluated by this method and validated by experiments. It should be noted that if a compressor does not experience forward spillage of tip leakage flow during its stall inception process, the axial momentum may not be the proper key indicator for its stability. Thus, this current control volume method may not applicable in such type of compressors.

\section{The integral control volume method}

\subsection{The Studied Compressor and Casing Treatments}

The Low-speed Axial Compressor of Institute of Engineering Thermophysics (IET-LAC) in authors' lab is adopted as the studied example. The investigated rotor is a single rotor compressor with 60 blades driven by an 18.5 $\mathrm{kW}$ AC motor at the design speed of $2400 \mathrm{rpm}$. The rotor has a 1.86 aspect ratio with a tip clearance $1.2 \mathrm{~mm}$. The schematic structure of the compressor and the measurement stations can be found in figure 2 of Ref. [7]. This rotor has been proven to be a tip-critical rotor and was used to demonstrate the single/double-groove casing treatments [7], tip-injection and other stall margin extension method.

Figure 2 depicts three typical end-wall treatment designs that are multiple circumferential grooves (CG5), skewed axial slots (SAS) and the self-injection (SELF-INJ). CG5 is composed with five circumferential grooves with a depth of
$6 \mathrm{~mm}$ and width of $3 \mathrm{~mm}$. The most upstream groove starts at $10 \% C_{a z}$. There is a $1 \mathrm{~mm}$ land gap between each two grooves. SAS is composed with equal axial slots extending in axial direction from the leading edge to trailing edge. Four slots lay right above each of the blade passage. The vertical depth is $7.5 \mathrm{~mm}$ for slot and the skew angle is $60^{\circ}$ aligned with the rotation. The SELF-INJ is designed as rotor tip casing recirculation with the idea of using suction and blowing to directly target tip leakage flow. The suction hole is locating at $100 \% C_{a z}$ downstream the blade trailing edge, bleeding the high pressure air with an angle of $45^{\circ}$ with the axial direction. The injector is on the right above of the leading edge and parallel with the axial direction for obtaining maximum axial bleeding velocity. In simulations, the injection angle is modified into 15 due to usage of the structure mesh requirement. There are fifteen self-injections are placed around the whole annulus in total.
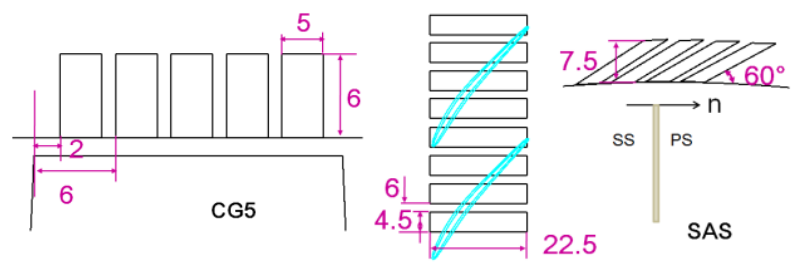

(a) multiple circumferential grooves, CG5 (b) skewed axial slots, SAS

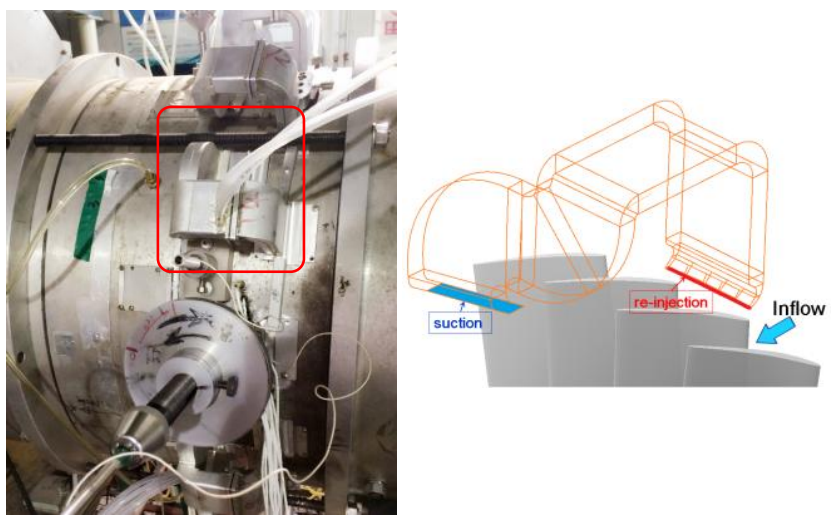

(c) self-injection casing treatment, SELF-INJ

Figure.2 The studied end-wall treatments

\subsection{The Numerical Method}

[In this sub-section, the highlighted sentences are newly included according to the reviewer 5\#'s suggestion.]

Single passage unsteady flow simulations were performed to predict the performance of this rotor without casing treatment. The computation code chosen for this research is EURANUS. Favre-Reynolds-averaged Navier-Stokes equations are discretized using a cell-centered explicit finite volume scheme according to Jameson in a relative coordinate system rotating with the reference frame. The system of equations is closed with the one-equation. Spalart-Allmaras turbulence model that represents a good 
balance between cost and precision according to [7] and was adopted in this study.

To ensure the computational results independent of grid distribution, the mesh used in this current study has been examined carefully by refining step by step. The detailed grid independence studies can be found in Ref. [12]. In order to evaluate properly the momentum fluxes at the tip region, the distance away from the wall of the first node had to be judiciously determined, and this minimum grid spacing gave $y+\leqslant 5$ at the walls. The rotor computational domain has a block-structured topology consisting of H-blocks for the inlet and outlet, a main O-block surrounding the rotor blade is used to model the rotor blade passage. Three sets of grids were refined one by one to ensure the independent grid. Finally, 173 grids are employed in the axial direction, of which 89 grid cells are set between the leading edge and trailing edge of the rotor. In the spanwise direction, 101 grids are employed, including 25 grid sets in the tip gap. This grid level inner tip gap is above the minimal recommended number in radial direction to guarantee the physics of the tip region according to Ref. [13]. The whole number of computational grids for this single passage is 1.01 million. Time resolved calculations were conducted to capture the unsteady flow at a near stall operating range. For the time-accurate investigations, the physical time of each time step is $1.436782 \mathrm{e}-5 \mathrm{~s}$ (30 time steps per blade passing period) and seudo-time iterations with a CFL number of 2-3 within each physical time step were performed. This methodology has proven to be able to capture the near stall unsteadiness by Lin et al. [9].

Figure. 3 (a) presents the calculated and measured performance curves for smooth casing. The definition of mass flow coefficient $\varphi$ and static to total pressure rise coefficient $\psi$ can be found in the Nomenclature table. The uniform inlet total pressure profile is applied as the inlet boundary condition while the radial momentum balance equation is used to obtain the exit static pressure distribution there. Mass flow coefficient and pressure rise coefficient derived from the simulation are calculated from these computational "probes" values using a processing method identical to those used to process the experimental data. From figure 3, although the predicted pressure performance is slightly lower than experimental data at near stall condition, the mass flow range shows a fairly good agreement over the whole investigated operating range. The flow coefficient $\varphi$ at stall onset is 0.496 with the maximum pressure rise coefficient $\psi$ of 0.441 judged from experiment for smooth casing, while, the calculated one is $\varphi=0.501$ with a $\psi=0.417$, which is considered as the last converged point of the calculation and is set as NS for subsequent comparison. It is noted that all of the measured performance curves are normalized by the standard inlet condition, with a total pressure of $101325 \mathrm{pa}$ and total temperature $288.15 \mathrm{~K}$. Figure 3 (b) and (c) following show the inlet and outlet axial velocity profiles with the simulated ones against the experimental results. The computed flow fields and experimental data also show good agreements in the local flow profiles (Fig. 3(b), (c)), especially for the rotor tip region. More details can be found in Ref. [12].

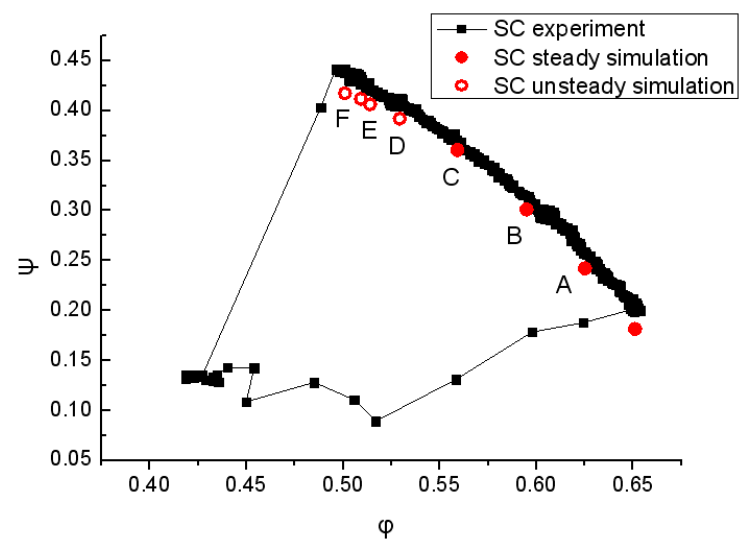

(a) The simulated and measured performance curves

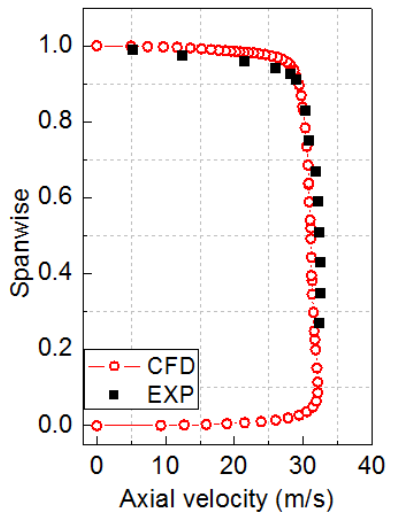

(b) inlet axial velocity profile at near stall point $(\varphi=0.501)$ for $\mathrm{SC}$

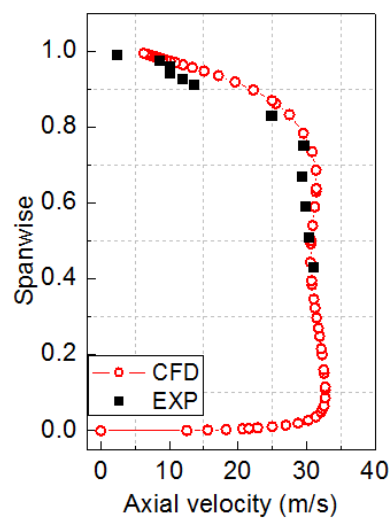

(b) outlet axial velocity profile at near stall point $(\varphi=0.501)$ for $\mathrm{SC}$
Figure.3 Comparison of calculation with measurement for IET-LAC, smooth casing

For capturing the interactions between the stationary casing treatments and the rotational blade passage, a sliding plane interface is utilized between the rotor and all of the casing treatments. With the lesson learn from the grid independence study for NASA R67 in Ref. [7], 31, 53, 61 points are employed in axial, tangential and radial direction for each of the grooves. The mesh density is kept the same for all casing treatment configurations. The smooth casing, circumferential and slots configurations were simulated by single passage simulation. For the self-injection case, it was simulated by four-passage simulation. There are totally 1.6 million, 1.4 million and 4.64 million for CG5, SAS and SELF-INJ for this simulation study respectively. 


\subsection{The control volumes and bell-shaped curves}

\subsubsection{The control volumes}

As mentioned in Introduction, the spillage of the MF/TLF interface out of the rotor tip leading edge plane is claimed as one of the criteria for spike stall inception in tip-critical axial compressors. However, the interfaces are generally observed as complex 3D surfaces in rotor reference frame, whose geometries and exact positions are difficult to identify. Moreover, the interface is essentially unsteady when compressor approaching to stall. Therefore, it is hard to justify which part of the interface would first swing out of leading edge and trigger to stall. It would be very difficult to correlate the position of the interface and its movement to compressor stability quantitatively. A method to indentify this interface in an integral of time- and spatialaveraged way is proposed in this paper.

Twenty control volumes are set at the rotor tip region. Among these control volumes, the first one starts at slightly upstream of the rotor leading edge, and the last one ends just at the trailing edge to cover the whole tip region. The width of each control volume is exactly half the width of the groove, in order to capture the injection-ejection flow mechanism observed by many researchers. These control volumes occupies one blade passage, containing the entire pitch. Periodic boundary conditions are applied to both of the control surfaces in this direction. In radial direction, the coverage of the control volumes needs to be carefully considered. To capture the balance of the axial momentum between MF and TLF, the flow features of TLF should be fully contained. The flow in the tip region, especially for the tip leakage flow, is recognized to be characterized by the complex vortices and shear flows with high shear stress and strong turbulence mixing. The entropy contours are thus utilized to distinguish the tip flow influence (Figure 4). The region with high entropy level at blade tip covers approximately $20 \%$ blade height from the casing, which indicates the TLF is not restrained in the tip gap but spreads into a certain radial depth. From this perspective, it is determined that the radial depth of these control volumes is from casing to $80 \%$ spanwise, which is approximately 20 times of the tip gap for this rotor. The way of setting the control volume's radial coverage is substantially different with Shabbir's [14] (concern about the momentum transport across the groove surfaces), Legras's [15] and Nolan's [16] (concern about the momentum transport with the tip gap and blade passage).

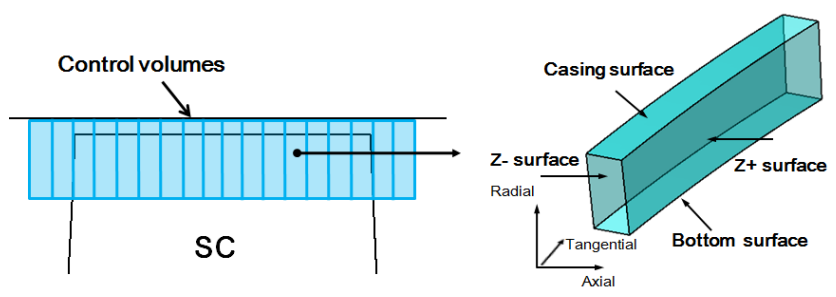

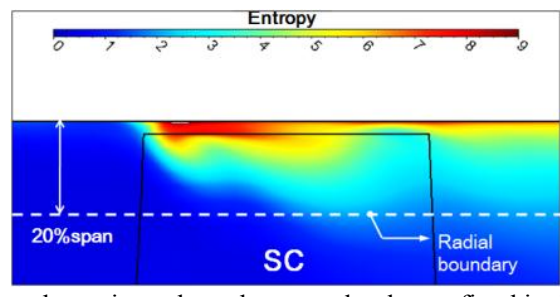

Figure.4 The schematic to show the control volumes fixed in the rotor reference frame and its axial, radial and tangential coverage.

\subsubsection{The bell-shaped curves}

The momentum equation is solved to quantify the axial momentum distribution within the tip region when the compressor is near the stall condition (operating point $\mathrm{F}$ labeled in Figure 3). The axial momentum of each control volume can be written as:

$$
\begin{aligned}
& \Sigma M_{z}=\int_{Z-} \rho V_{z}(\vec{V} \cdot \vec{n}) d A_{z-}+\int_{Z+} \rho V_{z}(\vec{V} \cdot \vec{n}) d A_{z+} \\
& +\int_{\mathrm{BT}} \rho V_{z}(\vec{V} \cdot \vec{n}) d A_{B T}+\int_{\mathrm{CS}} \rho V_{z}(\vec{V} \cdot \vec{n}) d A_{C S}
\end{aligned}
$$

Here, $M_{z}$ is the total axial momentum across each control volume. The subscripts Z-, Z+, CS and BT denote the four control surfaces that surrounding the control volume as shown in Figure 4. $\vec{V}$ is the absolute velocity vector and $\vec{n}$ is the surface unit normal. As for the single passage simulation, the forces on the two periodic boundary surfaces are neutralized with each other. The total axial momentum for each control volume element is the sum of the axial momentum across the four control surfaces, which is in fact the net axial force acting on that control volume.

Integrations are accomplished by a post-processing program. Figure.5 shows the local axial momentum for the smooth casing at $\varphi=0.501$, which is the near stall operating point for SC. The abscissa has been normalized by the tip axial chord. Here 0 represents the leading edge of the rotor tip while 1 represents the trailing edge. For the local axial momentum curve, there is a turning point appears at approximately $30 \% C_{a z}$. Before this point, the local axial momentum is positive, which means the flow shows a downstream tendency pushing by blade in pitch-averaged sense. This region is dominated by the main flow. After this point, the local axial momentum turns negative, which indicates that the flow is dominated by the reversed tip leakage flow and shows an opposite flow tendency. This turning point divides the rotor tip region into the main flow dominating region and the tip leakage flow dominating region in a pitch-wise sense. Here the values in Figure. 5 represent the whole annulus, which result from the single passage data multiplied by the number of the rotor blades. 


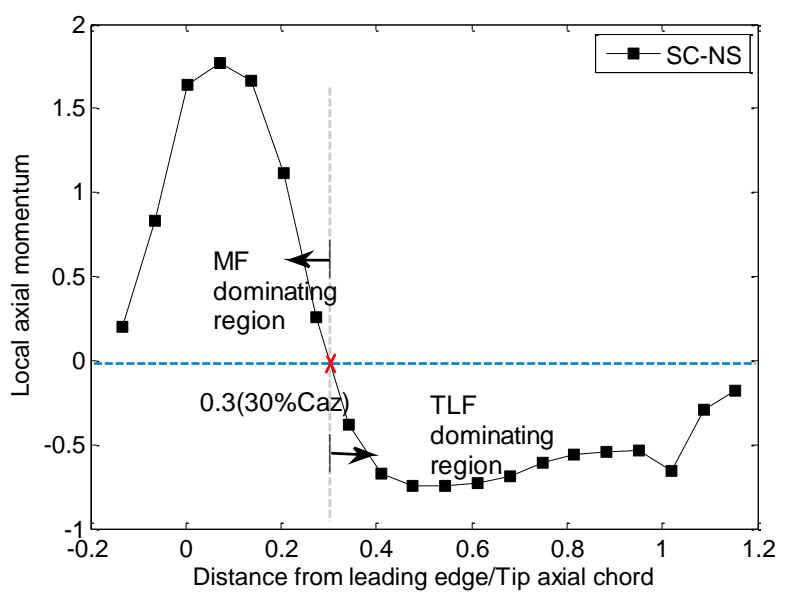

Figure.5 The local axial momentum distribution.

When adding the axial momentum for each control volume one by one, the cumulative axial momentum distribution for smooth casing is plotted as Figure.6. Such a cumulative axial momentum curve resembles a bell and it therefore named as the "bell-shape curve". An advantage of observing the axial momentum in an accumulative way is that the sensitivity of the axial width of the control volumes can be reduced, because each point on the curve is the total axial momentum of the region which covers from the first control volume to the local control volume, which is equal to the net axial force acting on the flow by this part. As marked with the red cross, a peak appears at $27 \% C_{a z}$ which divides this curve into two parts. Before reaching this peak, the slope of the curve is positive, which means every local control volume contributes positive axial momentum to the curve. This part is dominated by the main flow and corresponds that the blade pushes the flow downward in the sense of spatial-average. After the peak, the curve's slope turns negative, which means that the local control volumes begin to contribute negative axial momentum. It shows that the flow is dominated by the reversed TLF who flows against the main flow. This peak point represents the turning point that the local axial momentum changes from positive to negative. The dimensionless parameter $Z_{b p}$ is defined as the normalized axial location of the peak. It is a time- and spatial-averaged result to reflect the final balance between the main flow and TLF. It is worth noting that $Z_{b p}$ is the "static equilibrium point" as if we observe the interface in a timeand spatial- averaged way in space. It is not the exact location of the interface.

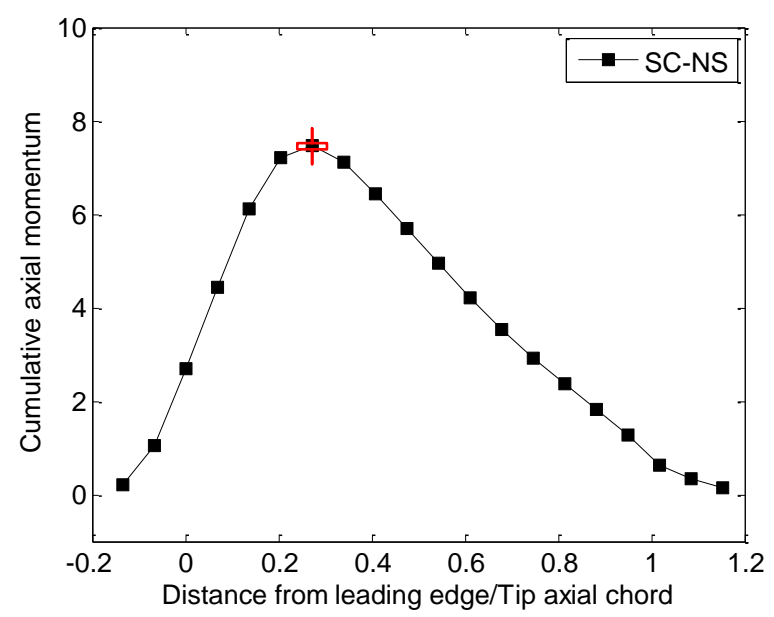

Figure.6 The cumulative axial momentum distribution (bell-shape curve) at NS for SC.

\subsubsection{The accuracy of the bell-shape curve}

The key parameter $Z_{b p}$ can indirectly evaluate the compressor stability. A question that naturally arises is that how accurate the bell-shaped curves is on evaluating the timeand spatial- averaged interface. The control volume methodology and the numerical scheme are discussed in this section.

Figure.7 shows a series of bell-shaped curves in the similar shape derived from varying the control volume height. When taking the tip gap (upper $2 \%$ annulus height) as the control volume, the cumulative axial momentum is much lower than those of which include a certain part of blade passage. The amplitude of the peak increases notably as the depth of control volume expanses from the tip gap (98\% span) to $95 \%$ span, while its growth slows down with the further increase of the control volume depth. Particularly, with an up to $5 \%$ blade height increase on the control volume depth (from $90 \%$ to $85 \%$ span), the peak is almost unchanged. Although the amplitude continually increases, the $Z_{b p}$ remains unchanged, which contributes the axial location of the "averaged interface" is insensitive to the increase of the control volume depth. It is indicated by this figure that as long as the majority of the TLF structure is involved, the axial location of the peak of bell-shaped curves can be reasonably guaranteed, which shows the robustness of this approach. 


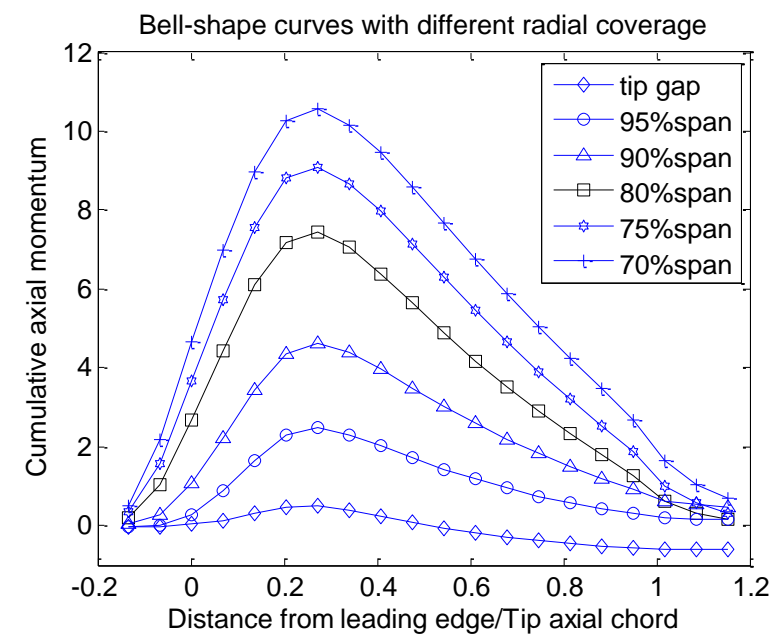

Figure.7 The dependency of bell-shaped curves on the control volume spanwise height, here $\mathrm{X} \%$ span means the radial coverage of the control volume is extending to $\mathrm{X} \%$ span from the casing. In this manner, tip gap is $98 \%$ span

The value of $Z_{b p}$ is influenced by the control volume width intrinsically. The bell-shaped curves with different control volume numbers are shown in Figure.8. Keep the domain of interest unchanged, the $Z_{b p}$ spatial resolution is increasing from $8 \% C_{a z}$ to $3 \% C_{a z}$ by refining the control volume numbers. With the control volume number increases from 15 to 40, their corresponding $Z_{b p}$ are changes as $24.0 \%$, $27 \%, 26.25 \%$ and $26.0 \% C_{a z}$ respectively. However, the shape of the curves and the amplitude of the peak are unchanged during this refining work. Although the control volume number increased doubled from 15 to 40 , the $Z_{b p}$ is only changed within $3 \% C_{a z}$. The impact of the control volume width on the evaluating SMI will be discussed in the later section.

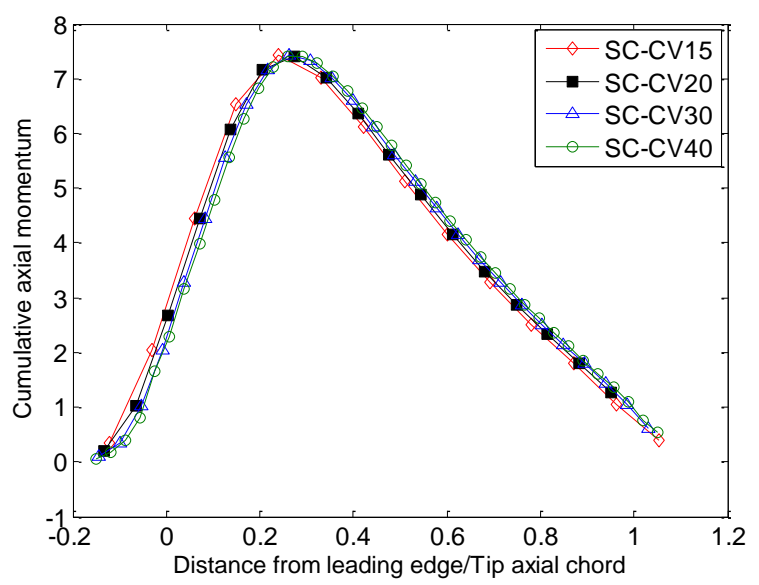

Figure. 8 The bell-shaped curves with different control volume width.

The significance of this method is that it can provide a preliminary judgment without calculating the whole overall performance curves. However, single-passage simulation was chosen as it can provide accurate enough prediction while keeping the computation time reasonably short for design purposes. Shortcomings of this approach are well understood when it comes to capture the cross-passage unsteady flows in stall inception process. The insensitivity of the bell-shaped curves on the single/multiple passage simulations is discussed.

Six-passage simulations at $\varphi=0.501$ is conducted. A periodic condition was applied at the circumferential boundaries of the first and sixth passage domain. Control volumes for this multi-passage simulation are set as Figure 9 shows, which are in the same manner with that of the single passage simulation in radial and axial directions. The only difference is the tangential coverage of the control volumes.

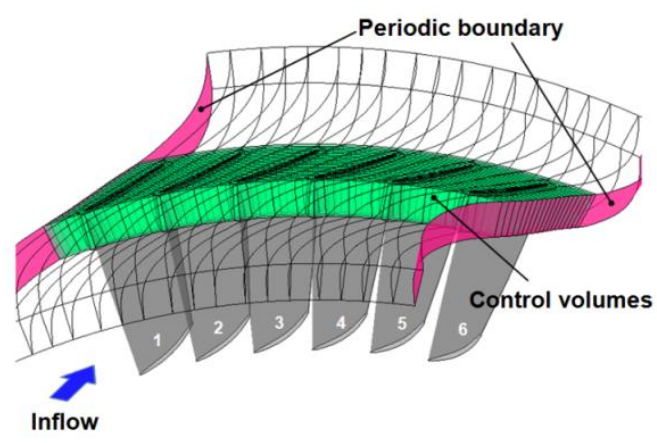

Figure.9 The control volumes for six passage simulation

The bell-shaped curves derived from single passage simulation (in black curve) and six passages simulation (in red curve) are compared in Figure 10. It is can be found that although the bell-shaped curves are not perfectly overlapped, they both maintain the same value of $Z_{b p}$, which confirms that the single passage simulation could suggest a rational $Z_{b p}$.

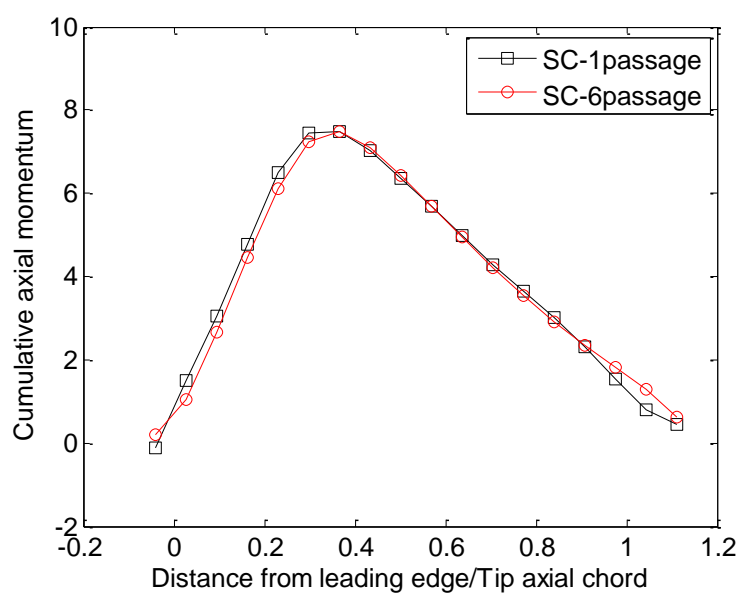

Figure. 10 The bell-shaped curves for single passage simulation and multiple passage simulation for smooth casing

\subsubsection{The bell-shaped curves during throttling process}

The variations of bell-shaped curves during the throt- 
tling for this low-speed compressor are investigated in Figure 11. The studied operating points A-F have been labelled in Fig. 3(a) previous. The peak of bell-shaped curves continually shifts towards the blade leading edge as the mass flow rate decreases. During this throttling process, the blade tip loading is changing which result in an updating balance between the main flow and the TLF. The shrinking of the main flow dominating region and the expanding of the TLF dominating region reflect the fact that the MF/TLF interface moves upstream with the decrement of the mass flow for both cases.

Figure 11(b) collects the crucial parameter $Z_{b p}$ as a function of mass flow rate for both the smooth casing. As the mass flow coefficient throttling from 0.625 to 0.501 , the $Z_{b p}$ decreases from 0.6 to 0.27 . The $Z_{b p}$ continually decreases as the compressors approach to stall. This movement of the parameter $Z_{b p}$ during throttling process conforms well to the experimental findings by Cameron et al. [10] on a transonic compressor. From this perspective, although it is hardly to identify the position due to the highly three dimensional TLF, the axial location of the bell-shape curve peak can be deemed as the key flow parameter to correlate the position of MF/TLF interface and further to indirectly evaluate the compressor stability. Note that the bell-shaped curves shown in the figure are the time-averaged results for one fluctuation period for operating points D-F.

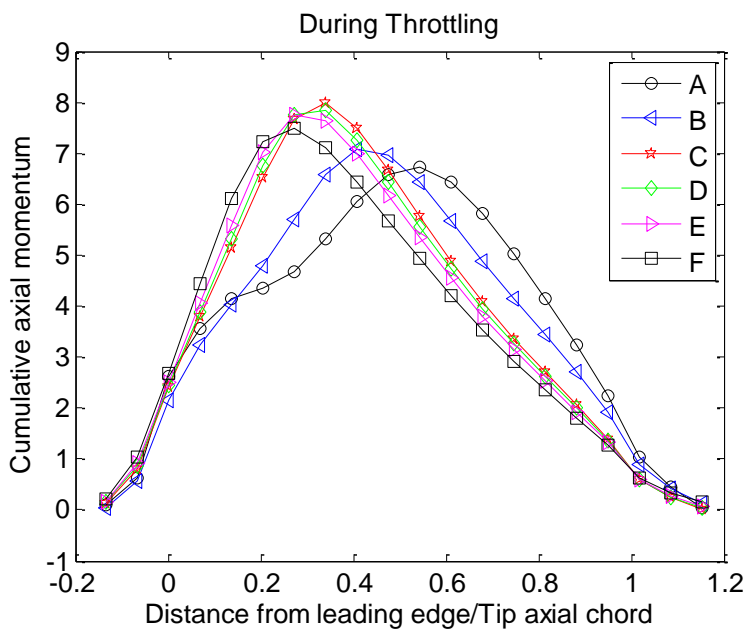

(a) Bell-shaped curves correspond to the operating points labeled in Figure.3 (a)

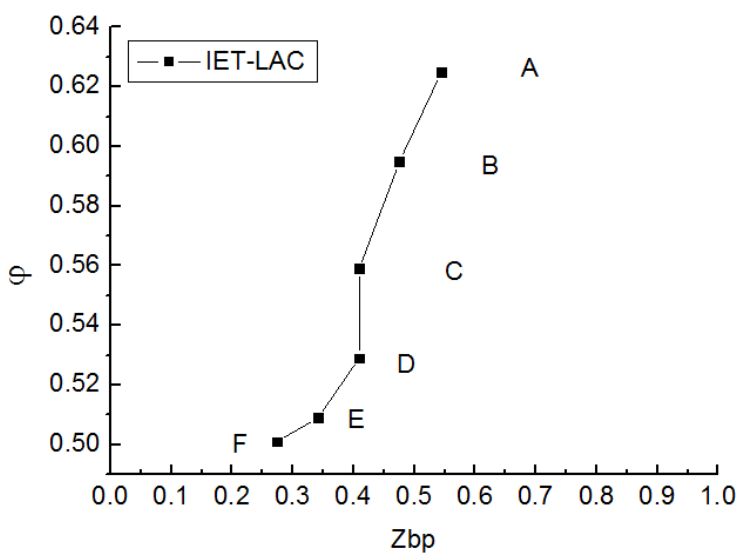

(b) The $Z_{b p}$ for collected for the operating points labeled in Figure.3 (a)

Figure.11 The variation of the bell-shaped curves during the compressor approach to stall.

\section{Evaluation of the effectiveness of the casing treatments by the integral method}

\section{[This section has been totally revised]}

The integral control volume method is then employed to evaluate the effectiveness of the three studied casing treatments without capturing the entire performance curves. The analytical steps can be estimated as follows:

Step 1. Perform simulations for the three end-wall casing treatment configurations at the mass flow coefficient of the near stall for $\mathrm{SC}(\varphi=0.501)$;

Step 2. Set the rotor tip control volumes for these configurations as illustrated in section 2.3.1. Note that the control volumes are set at the rotor tip region, the casing treatment domains are not included in.

Step 3. Calculate the axial momentums for the control volumes and then adding these values accumulatively.

Step 4. Obtain the bell-shaped curves and evaluate effectiveness by comparing the $Z_{b p}$ for those curves.

The bell-shaped curves for the three studied end-wall treatments are plotted in Figure 12. Compared to SC, all of the casing treatments have a more downstream $Z_{b p}$ than $\mathrm{SC}$, which means they all beneficial to the compressor stall limit. It is suggested by the bell-shaped curves that the SAS has the highest capability on stall enhancing, with $Z_{b p}$ of 0.544. The multiple grooves configuration CG5 has a $Z_{b p}$ of 0.409 , shows a medium capability on stall margin improvement. The $Z_{b p}$ of SELF-INJ is 0.34 , which indicates that it is less effective than the other two casings.

Among the three casing treatment configurations, the slot-type and self-injection type of casing treatments uplift the left branch of the bell-shape curve, while the groove-type shows an opposite tendency. It is can be inferred that although the MF/TLF interface is pushed downstream by all of the three typical treated casings, the flow mechanism by the casing treatments could be different. 


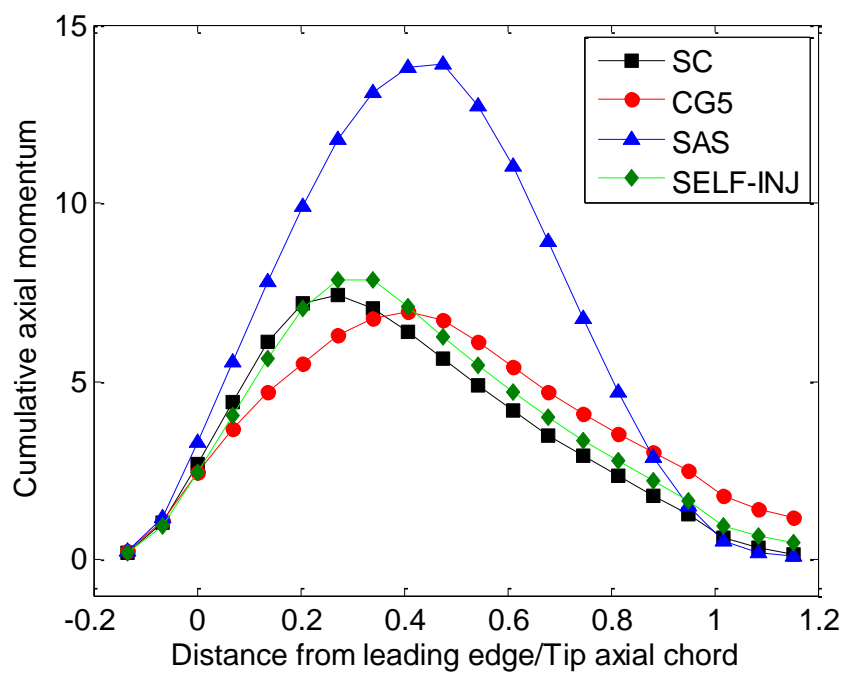

Figure.12 The bell-shaped curves for the studied casing treatments.

Up to now, we have quickly compared the effectiveness of the three casing treatment with only calculating the flow field at one single operating point. Here, one of a previous experimental measurement on a grooved casing treatment named as CG13 is recalled to preliminary estimate the SMI levels of the three studied casings. CG13 is a double-groove configuration with a combination of two single grooves of the same width and depth as CG5. Its specifics can be found in Ref. [7]. Figure 13 shows the bell-shaped curves and the previous experimental performance curves for CG13 and SC. Note that the tip clearance is $0.8 \mathrm{~mm}$ for these two casings. From the results shown in Figure 13, the CG13 has a $Z_{b p}$ of $27.4 \% C_{a z}$ and SC has a $Z_{b p}$ of $21 \% C_{a z}$, while CG13 generates $5.28 \%$ in SMI.

As a reference, CG13 obtains an increase in $Z_{b p}$ for 0.064 compared to SC, which means the $5.28 \%$ in SMI is contributed by the downstream-pushed spatial averaged interface with $6.4 \% C_{a z}$. According to this, SELF-INJ has the equal increase in $Z_{b p}$ with CG13, which is supposed to exhibit the same SMI level as CG13. The multiple-groove configuration CG5 might generate at least $10 \%$ in SMI. For the most favorable configuration, SAS sustains the most downstream of peak point, which is predicted to obtain an SMI that higher than $15 \%$.

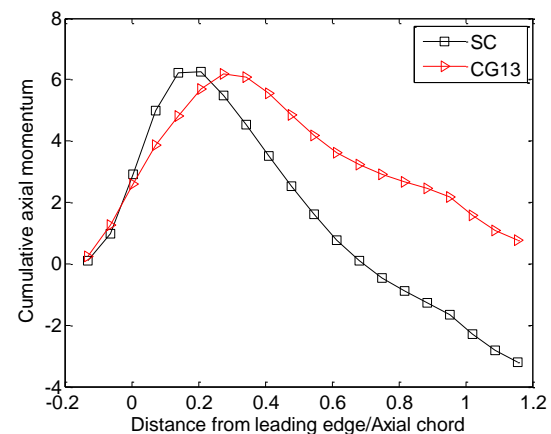

(a) The bell-shaped curves for SC and a double groove scheme CG13 [7]

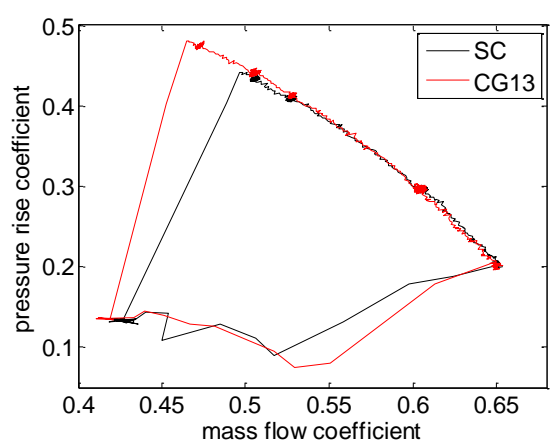

(b) The experimental performance curves [7]

Figure. 13 Bell-shaped curves for SC and CG13 and the measured performance curves by author's previous work on IET-LAC [7]. Note that the tip clearance for these two casings is $0.8 \mathrm{~mm}$, so the bell-shaped curve is of slightly difference with that of the three studied casing treatments.

Experiments are carried out to verify these assessments. The measured performance curves for the treated and untreated casings are given in Figure 14(a) and (b). Their SMI values are following summarized in Table 1 . Delta $Z_{b p}$ in Table 1 is defined as the $Z_{b p}$ increase with the treated casings compared to SC. The trends on SMI for the casing treatments agree well with the assessments with the bell-shaped curves. The SAS configuration receives the highest SMI, with $27.4 \%$, while the self-injection configuration the lowest, with $5.26 \%$. With the same value of delta $Z_{b p}$ as CG13, the SMI are almost equal with these two casings. Also, the $Z_{b p}$ for CG5 is two times than that of SELF-INJ, whose SMI turns to be twice than that of SELF-INJ.

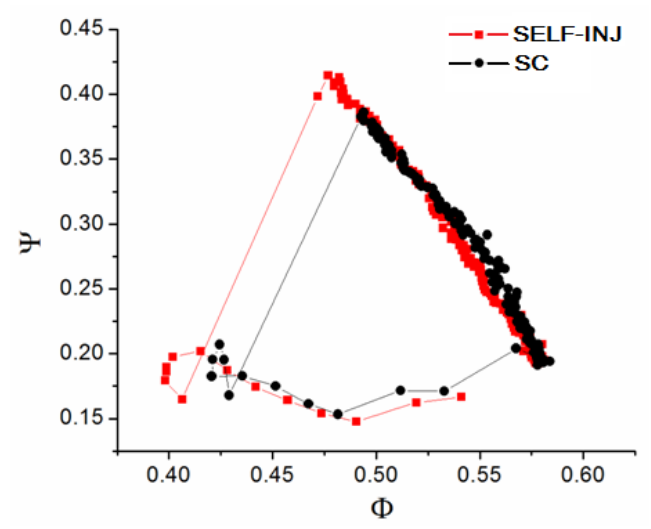

(a) Experimental performance curves with SELF-INJ compared with SC 


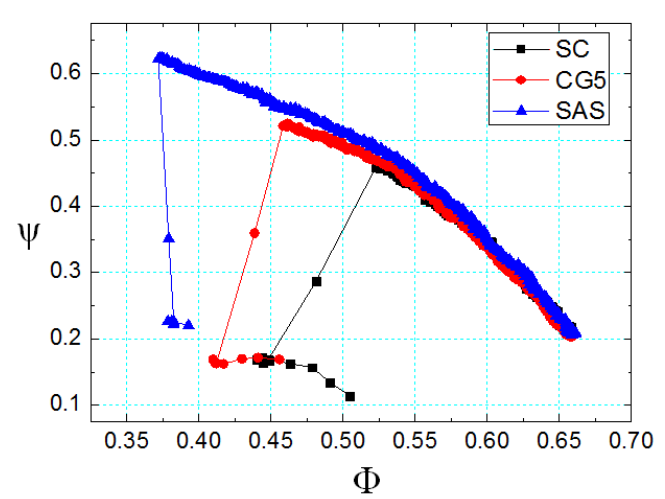

(b) Experimental performance curves with CG5 and SAS compared with $\mathrm{SC}$

Figure.14 Measured performance curves for self-injection and smooth casing.

Table 1 The $Z_{b p}$ from control volume method and the experimental SMI

\begin{tabular}{cccc}
\hline End-wall treatment & $Z_{b p}$ & $\Delta Z_{b p}$ & Experimental SMI \\
\hline SC & 0.273 & - & - \\
SELF-INJ & 0.341 & 0.068 & $5.26 \%$ \\
Double-groove CG13[14] & 0.274 & 0.064 & $5.28 \%$ \\
CG5 & 0.409 & 0.136 & $10.8 \%$ \\
SAS & 0.544 & 0.271 & $27.4 \%$ \\
\hline
\end{tabular}

Note that by now on, the effectiveness of various casing treatments can be achieved by this integral method, but it is still a challenge to predict the accurate SMI value directly by this method. In this paper, the prediction in SMI according to an existed double-groove measurement is based on simply assuming that SMI is linear with $Z_{b p}$. However, the tested results reveal that the $Z_{b p}$-SMI trend is nonlinear. This resulted in an under-estimated SMI of SAS compared with other configurations. It is reasonable because the upstream movement of the interface between the main flow and tip leakage flow is not a uniform motion during the throttling (see figure 11(b)). If we want to further predicting the exact SMI value by this method directly, a few known cases with experimental SMI data may help to calibrate the predicting in SMI value for new casing configurations. The measured accuracy on SMI is $0.68 \%$. Note again that the tip clearance of CG13 is $0.8 \mathrm{~mm}$, which result in a slightly different bell-shape curve compared with the casings studied in this current paper.

\section{Conclusion}

Based on the understanding on the stall mechanism of tip-critical axial compressors, a novel post-computational method is proposed and applied to assess the effectiveness of the three typical end-wall treatments in this paper. Conclusions can be summarized as following:

1) An integral method is developed to assess the efficacy of the casing treatment without calculating the entire per- formance curves. The basic idea of this method is to compare the time- and spatial- averaged locations of the MF/TLF interfaces among various casing treatment configurations at the near stall point of the smooth casing. This interface is viewed as an integral effect of the axial momentum balance between the main incoming flow and the tip reversed flow.

2) Cumulative axial momentum distribution of the tip region named as bell-shaped curves is proposed to correlate the mechanism of flow field with the compressor stability. The axial location of the peak $\left(Z_{b p}\right)$ is considered as the spatial- and time-averaged axial location of the complex 3D MF/TLF interface. The $Z_{b p}$ decreases during the throttling, which indicates the interface moves towards rotor leading edge when the compressor approaches to stall.

3) The accuracy of the bell-shaped curves is discussed. It is found the height, width of integral regions are insensitive with $Z_{b p}$. Multi- and single- passage simulations are also discussed to ensure the single passage simulation can suggest a rational $Z_{b p}$ value with engineering accuracy.

4) Three different types of typical casing treatments are assessed by the bell-shaped curves. Their SMI values are further predicted through a comparison of $Z_{b p}$ value with a known SMI configuration.

5) Experiments are conducted to verify the assessments. The measured SMI trends are consistent with the assessment, which confirms this method not only can compare the effectiveness for various casing treatments, but also can provide a preliminary assessment on SMI.

By simply simulating the operating points at near stall mass flow rate for smooth casing, and comparing the bell shape curves, the efficacy of the candidates of grooved casings can be easily assessed in a short time. This method is quite different from the common approaches that calculate the stall margin by numerically capturing the whole performance curve. It is well recognized that many difficult challenges still exist in capturing the experimental stall point with current numerical technologies [17]. Highly refined meshes, sophisticated turbulence model, full-annulus computation domain, etc. would be needed in order to accurately capture the unsteady stalling processes. The proposed approach avoids these difficulties by concentrating the numerical efforts on the near stall points in which the flow physics would be much simpler than the stall inceptions. This simplification, plus the post-CFD data analysis with control volume approach, significantly reduces the requirements on the numerical schemes, and thus make it possible to quickly screening a variety of casing treatments before involving high-fidelity, full-annulus and time-accurate simulations with highly sophisticated turbulence models. The reliability of the proposed approach lies on the hypothesis that the spike stall precursors could result from the leading edge spillage of the MF/TLF interface, which has been and continue being tested in the compressor research communi- 
ty. Although this paper is concentrated on isolated rotors, the authors are confident that it should work in multi-row or multi-stage environment which exhibit the similar tip-sensitive stalling behavior.

The authors plan to continue refine the method. In a short term, more sophisticated numerical schemes will be

This work is supported by National Science Foundation of China with project No. 51176188 and No.51306178.

1 Hathaway M.D.2007, "Passive end-wall treatments for enhancing stability". NASA TM-214409.

2 Bailey E.E. 1984, "Effect of grooved casing treatment on the flow range capability of a single-stage axial-flow compressor." NASATM-2459.

3 Takata H.,Tsukuda Y. 1977,"Stall margin improvement by casing treatment-its mechanism and effectiveness".ASME Journal of Engineering for Power, 99(1): 121-133.

4 Gelmedov F.Sh., MileshinV.I.,Kozhemyako,P.G. Orekhov, I.K., 2014, "Stall Margin Improvement in Three-Stage Low Pressure Compressor by use of Slot type Casing Treatment", ASME paper, GT2014-26298.

5 Fujita H.,Takata H., 1984, "A study on configurations of casing treatment for axial flow compressors", Bulletin of JSME, 27(230): 1675-1681.

6 Hah C., Muller M., Schiffer H-P, 2012,"Study of Convective Flow Effects in End-wall Casing Treatments in Transonic Compressor Rotors", ASME paper, GT2012-68411.

7 Nan X., Lin F., Wang S., 2014,"The Budget Analysis of Axial Momentum of the Rotor Tip Flows for Axial Compressors with Circumferential Grooves", ASME Paper, GT2014-26495.

8 Vo H. D., Tan C. S., and Greitzer E. M., 2008, "Criteria for Spike Initiated Rotating Stall," ASME Journal of Turbomachinery, 130, p. 011023. explored to capture the entropy production in order to perform efficiency analysis for casing treatments. In a long term, several sub-modules, such as the selection of the casing treatment geometry, the auto grid generation, as well as the efficiency assessment will be integrated to form an automatic optimization analysis platform.

9 Lin F., Zhang J., Chen J., and Nie C., 2008, "Flow Structure of Short-Length-Scale Disturbance in an Axial-Flow Compressor," Journal of Propulsion and Power, 24, pp. 1301-1308.

10 Cameron J.D., Benningtion M.A., Ross M.H., 2013, "The Influence of Tip Clearance Momentum Flux on Stall Inception in a High-Speed Axial Compressor", ASME Journal of Turbomachinery, Vol. 135(5), p.051005.

11 Erler E., Vo H.D., Yu H., "Desensitization of axial compressor performance and stability to tip clearance flow". ASME paper, 2015, GT2015-42746.

12 Nan X. 2014, "The Compressor Rotor Tip Control Volume Method and it's Application on Circumferential Groove Casing Treatment". Institute of Engineering Thermophysics, Chinese Academic of Sciences.PhD thesis, China

13 Erler E., Vo H.D, Yu H, "Desensitization of Axial Compressor Performance and Stability to Tip Clearance Size", ASME paper GT2015-42746.

14 Shabbir A., Adamczyk J.J.,2005, "Flow mechanism for stall margin improvement due to circumferential casing grooves on axial compressors", ASME Journal of Turbomachinery, Vol. 127(3), p708-171.

15 Legras G., Gourdain N., et.al., 2014, "Analysis of Unsteadiness on Casing Treatment Mechanisms in an Axial Compressor", ASME paper, GT2011-45806.

16 Nolan S., 2005, "Effect of radial transport on compressor tip flow structures and enhancement of stable flow range", Master thesis, Massachusetts Institute of Technology, USA.

17 Houghton, T., Day,I., 2009, "Enhancing the stability of the subsonic compressor using casing grooves", ASME Paper, GT2009-59210. 